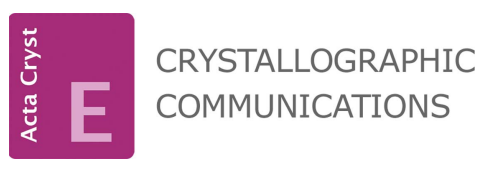

ISSN 2056-9890

Received 13 November 2019

Accepted 5 December 2019

Edited by H. Ishida, Okayama University, Japan

Keywords: crystal structure; $E$ configuration; iodophenyl ring; chalcone; (E)-3-(3-iodophenyl)1-(4-iodophenyl)prop-2-en-1-one.

CCDC reference: 1970266

Supporting information: this article has supporting information at journals.iucr.org/e

\section{Crystal structure and Hirshfeld surface analysis of (E)-3-(3-iodophenyl)-1-(4-iodophenyl)prop-2-en-1- one}

Kieran J. Spruce, ${ }^{a}$ Charlie L. Hall, ${ }^{\mathrm{a} *}$ Jason Potticary, ${ }^{\mathrm{a}}$ Natalie E. Pridmore, ${ }^{\mathrm{a}}$ Matthew E. Cremeens, ${ }^{b}$ Gemma D. D'ambruoso,, Masaomi Matsumoto, Gabrielle I. Warren, ${ }^{b}$ Stephen D. Warren ${ }^{b}$ and Simon R. Hall ${ }^{a}$

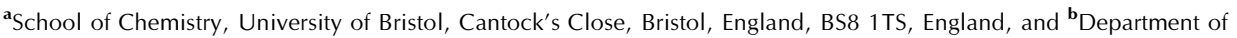
Chemistry \& Biochemistry, Gonzaga University, 502 E Boone Ave, Spokane, WA 99258, USA. *Correspondence e-mail: simon.hall@bristol.ac.uk
}

The title compound, $\mathrm{C}_{15} \mathrm{H}_{10} \mathrm{I}_{2} \mathrm{O}$, is a halogenated chalcone formed from two iodine substituted rings, one para-substituted and the other meta-substituted, linked through a prop-2-en-1-one spacer. In the molecule, the mean planes of the 3-iodophenyl and the 4-iodophenyl groups are twisted by $46.51(15)^{\circ}$. The calculated electrostatic potential surfaces show the presence of $\sigma$-holes on both substituted iodines. In the crystal, the molecules are linked through type II halogen bonds, forming a sheet structure parallel to the $b c$ plane. Between the sheets, weak intermolecular $\mathrm{C}-\mathrm{H} \cdots \pi$ interactions are observed. Hirshfeld surface analysis showed that the most significant contacts in the structure are C $\cdots \mathrm{H} / \mathrm{H} \cdots \mathrm{C}(31.9 \%)$, followed by $\mathrm{H} \cdots \mathrm{H}(21.4 \%), \mathrm{I} \cdots \mathrm{H} / \mathrm{H} \cdots \mathrm{I}(18.4 \%)$. $\cdots \mathrm{I}$ $(14.5 \%)$ and $\mathrm{O} \cdots \mathrm{H} / \mathrm{H} \cdots \mathrm{O}(8.1 \%)$.

\section{Chemical context}

Chalcones are aromatic ketones which have shown potential as antibacterial, antifungal and anti-inflammatory agents (D'silva et al., 2011). These molecules are essential to the biosynthesis of flavonoids through a conjugate ring-closure to form flavone and have also attracted attention for their potential use in opto- and organic electronics (Shetty et al., 2016, 2017). As a family of molecules, substituted chalcones can be readily synthesized via a Claisen-Schmidt condensation reaction between an appropriately functionalized acetophenone and benzaldehyde. Substitutions on each of the benzene rings are currently being investigated in order to interrogate how the electronic properties of the crystal are altered. The iodo-substituted rings present in the title compound allows for the formation of iodine channels in the crystal, a conformation which may afford a change in the crystal's electrical properties.<smiles>O=C(/C=C/c1cccc(I)c1)c1ccc(I)cc1</smiles>

\section{Structural commentary}

The title compound comprises two aromatic rings, 4-iodophenyl (1-Ring) and 3-iodophenyl (3-Ring), which are 


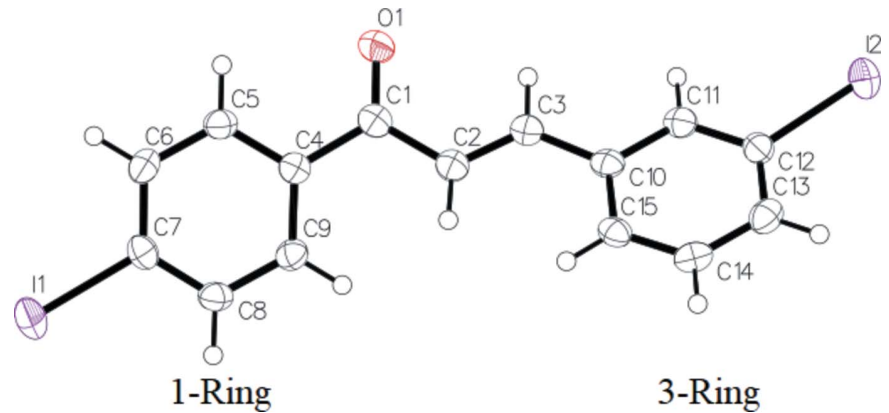

Figure 1

The molecular structure of the title compound, showing the atom labelling and displacement ellipsoids drawn at the $50 \%$ probability level.

connected, respectively, to atoms $\mathrm{C} 1$ and $\mathrm{C} 3$ of the $-\mathrm{CO}-$ $\mathrm{CH}=\mathrm{CH}$ - enone bridge (Fig. 1). The backbone torsion angles are $\mathrm{C} 5-\mathrm{C} 4-\mathrm{C} 1-\mathrm{C} 2=151.6(4)^{\circ}, \mathrm{C} 4-\mathrm{C} 1-\mathrm{C} 2-\mathrm{C} 3=$ $171.9(4)^{\circ}, \mathrm{C} 1-\mathrm{C} 2-\mathrm{C} 3-\mathrm{C} 10=176.4(4)^{\circ}$ and $\mathrm{C} 2-\mathrm{C} 3-$ $\mathrm{C} 10-\mathrm{C} 11=170.4(5)^{\circ}$. The mean planes of the 3-iodophenyl and 4-iodophenyl groups are twisted by $46.51(15)^{\circ}$ relative to each other. The $\mathrm{H}$ atoms of the propenone group are transconfigured.

\section{Supramolecular features}

Electrostatic potential surfaces [Fig. 2(a)-(c)] show the presence of $\sigma$-holes on both substituted iodines, I1 and I2, which allow for halogen bonding of a bifurcated type II. Partial packing diagrams are shown in Fig. 3(a)-(c). Interestingly, these halogen bonds form exclusively between equivalent iodine atoms, either para-para or meta-meta. The geometries of the halogen bonds are $\mathrm{I} 1 \cdots \mathrm{I} 1^{\text {iv }}=4.0980$ (9) $\AA$,

$-0.02 \mathrm{e} / \AA$

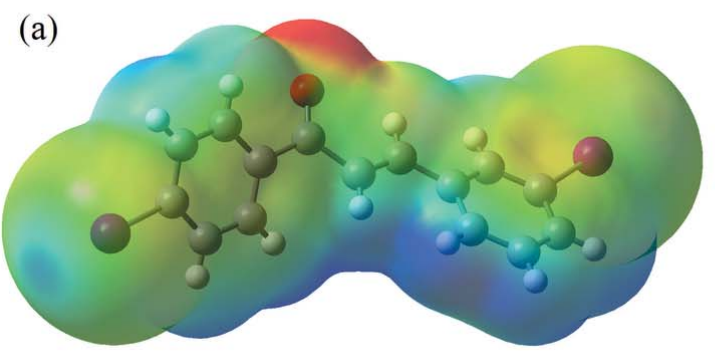

(b)

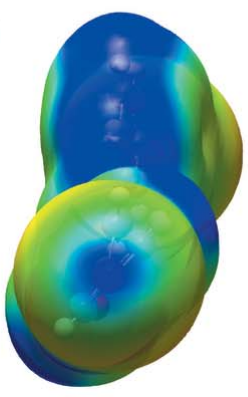

(c)

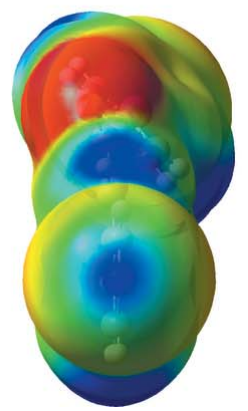

Figure 2

Electrostatic potential mapped onto an electron density isosurface with isovalue $0.02 \mathrm{e}^{-3}$, calculated using B3LYP at the LANL2DZ level. Red and blue regions show negative and positive electric potentials, respectively. $(a)$ shows the potential of the substituted chalcone molecule. $(b)$ and $(c)$ show the $\sigma$-holes on 1-Ring and 3-Ring, respectively.

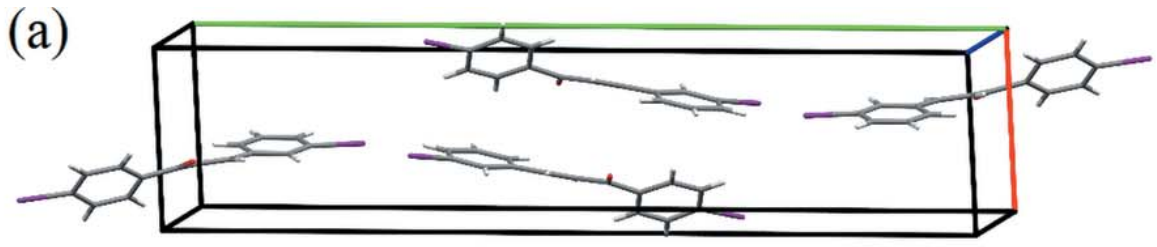

(b)

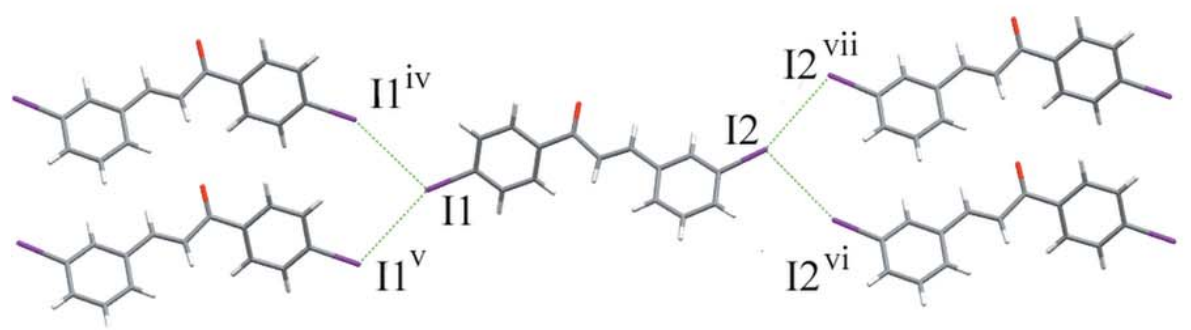

(c)

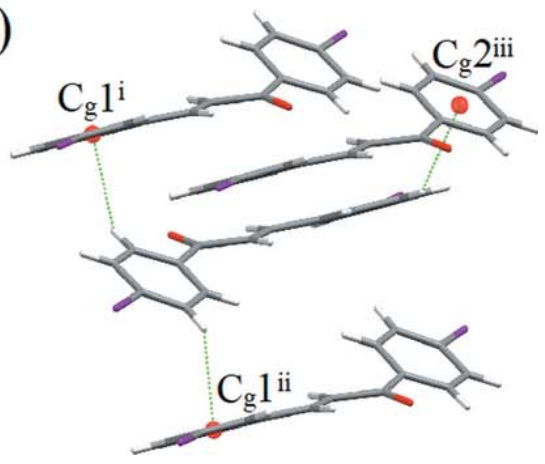

Figure 3

(a) A packing diagram of the title compound in the unit cell. Red, green and blue axes indicate $a, b$ and $c$, respectively. ( $b$ ) Meta-meta and para-para halogen bonds indicated by dashed lines. (c) Three weak $\mathrm{C}-\mathrm{H} \cdots \pi$ interactions (dashed lines; $\mathrm{C} 5-\mathrm{H} 5 \cdots C g 1^{\mathrm{i}}, \mathrm{C} 8-\mathrm{H} 8 \cdots C g 1^{\mathrm{ii}}$ and $\mathrm{C} 14-\mathrm{H} 14 \cdots C g 2^{\mathrm{iii}}$ ). $C g 1$ and $C g 2$ are the centroids of the $\mathrm{C} 10-\mathrm{C} 15$ and $\mathrm{C} 4-\mathrm{C} 9$ rings, respectively. [Symmetry codes: (i) $1-x, 1-y, 1-z ;$ (ii) $2-x, 1-y, 2-z ;$ (iii) $1-x$, $1-y, 2-z$; (iv) $x, \frac{1}{2}-y,-\frac{1}{2}+z$; (v) $x, \frac{1}{2}-y, \frac{1}{2}+z$; (vi) $x, \frac{3}{2}-y, \frac{1}{2}+z$; (vii) $x, \frac{3}{2}-y,-\frac{1}{2}+z$. .] 
Table 1

Hydrogen-bond geometry $\left(\AA,^{\circ}\right)$.

$C g 1$ and $C g 2$ are the centroids of the $\mathrm{C} 10-\mathrm{C} 15$ and $\mathrm{C} 4-\mathrm{C} 9$ rings, respectively.

\begin{tabular}{lllll}
\hline$D-\mathrm{H} \cdots A$ & $D-\mathrm{H}$ & $\mathrm{H} \cdots A$ & $D \cdots A$ & $D-\mathrm{H} \cdots A$ \\
\hline $\mathrm{C} 5-\mathrm{H} 5 \cdots C g 1^{\mathrm{i}}$ & 0.95 & 2.78 & $3.406(5)$ & 124 \\
$\mathrm{C} 8-\mathrm{H} 8 \cdots C g 1^{\mathrm{ii}}$ & 0.95 & 2.85 & $3.491(5)$ & 126 \\
$\mathrm{C} 14-\mathrm{H} 14 \cdots C g 2^{\mathrm{iii}}$ & 0.95 & 2.77 & $3.440(5)$ & 129 \\
\hline
\end{tabular}

Symmetry codes: (i) $-x+1,-y+1,-z+1$; $\quad$ (ii) $-x+2,-y+1,-z+2$; (iii) $-x+1,-y+1,-z+2$.

$\mathrm{C} 7-\mathrm{I} 1 \cdots \mathrm{I} 1^{\mathrm{iv}}=113.85(13)^{\circ}, \mathrm{I} 1 \cdots \mathrm{I} 1^{\mathrm{v}}=4.0980(9) \AA$ and $\mathrm{C} 7-$ $\mathrm{I} \cdots \mathrm{I} 1^{\mathrm{v}}=154.47(13)^{\circ}$ for the para-para $\mathrm{I} \cdots \mathrm{I}$ bonds, and $\mathrm{I} 2 \cdots \mathrm{I} 2^{\mathrm{vi}}=3.9805(8) \AA, \quad \mathrm{C} 12-\mathrm{I} 2 \cdots \mathrm{I} 2^{\mathrm{vi}}=108.20(13)^{\circ}$, $\mathrm{I} 2 \cdots \mathrm{I} 2^{\text {vii }}=3.9805(8) \AA$ and $\mathrm{C} 12-\mathrm{I} 2 \cdots \mathrm{I} 2^{\text {vii }}=157.30(13)^{\circ}$ for the meta-meta I $\cdots$ I bonds [symmetry codes: (iv) $x, \frac{1}{2}-y$, $-\frac{1}{2}+z$; (v) $x, \frac{1}{2}-y, \frac{1}{2}+z$; (vi) $x, \frac{3}{2}-y, \frac{1}{2}+z$; (vii) $x, \frac{3}{2}-y,-\frac{1}{2}+z$. A sheet structure is formed parallel to the $b c$ plane. There are also three weak $\mathrm{C}-\mathrm{H} \cdots \pi$ interactions (Table 1 ) between the sheets.

Hirshfeld surfaces, mapped over $d_{\text {norm }}$, shape-index and $d_{\mathrm{e}}$, and two-dimensional fingerprint plots of the title compound were calculated using CrystalExplorer17.5 (Turner et al., 2017). Hirshfeld surfaces [Fig. 4(a) and (c)] highlight the relationship between the contact distance and the van der Waals radii (Venkatesan et al., 2016). The Hirshfeld surface mapped over the shape-index [Fig. 4(b)] shows depressions on both 1-Ring and 3-Ring, which is indicative of $\mathrm{C}-\mathrm{H} \cdots \pi$ interactions. Twodimensional fingerprint plots are used to illustrate the intermolecular contacts between molecules within the crystal structure. The fingerprint plots of all significant interactions are shown in Fig. 5(a)-(f). C $\cdots \mathrm{H} / \mathrm{H} \cdots \mathrm{C}$ contacts [Fig. 5(b)]
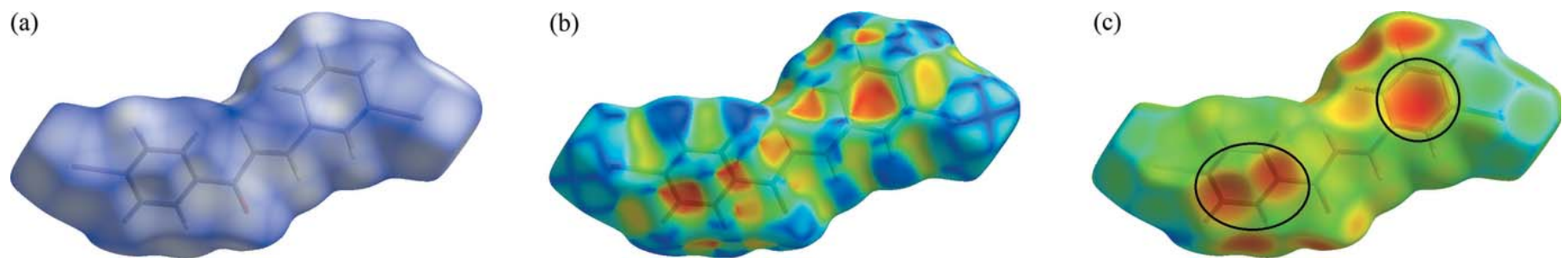

Figure 4

Hirshfeld surfaces of the title compound, mapped with $(a) d_{\text {norm }}$, where white regions represent interactions equal to, and blue regions represent interactions shorter than the sum of their van der Waals radii, $(b)$ the shape-index, and $(c) d_{\mathrm{e}}$, where the circled areas indicate the $\mathrm{C}-\mathrm{H} \cdots \pi$ interactions.

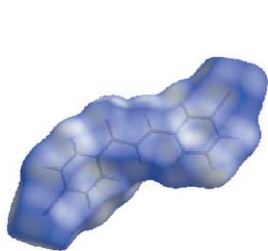

(a) All --- All 100\%

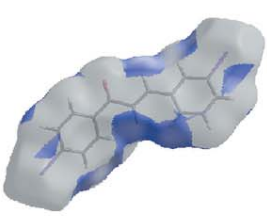

(c) $\mathrm{H}---\mathrm{H} 21.4 \%$

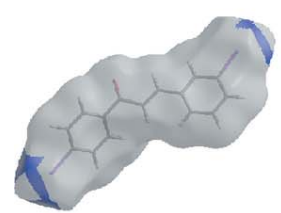

(e) I---I $14.5 \%$
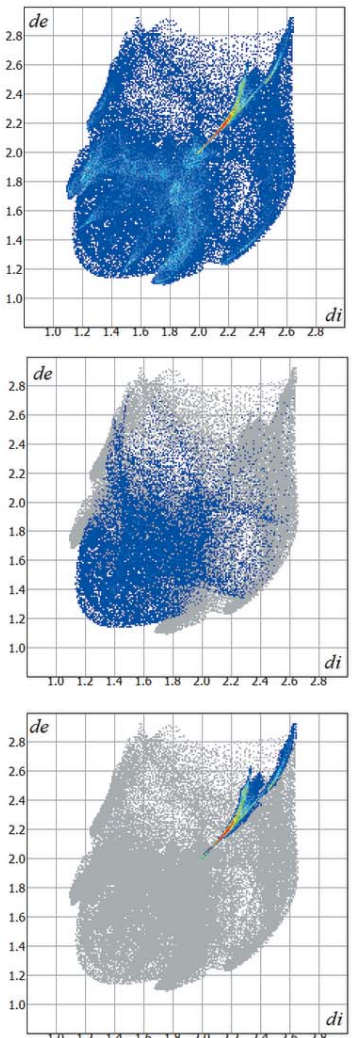

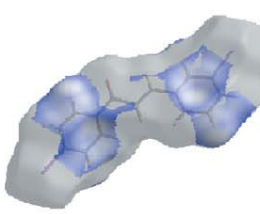

(b) C---H/H---C $31.9 \%$
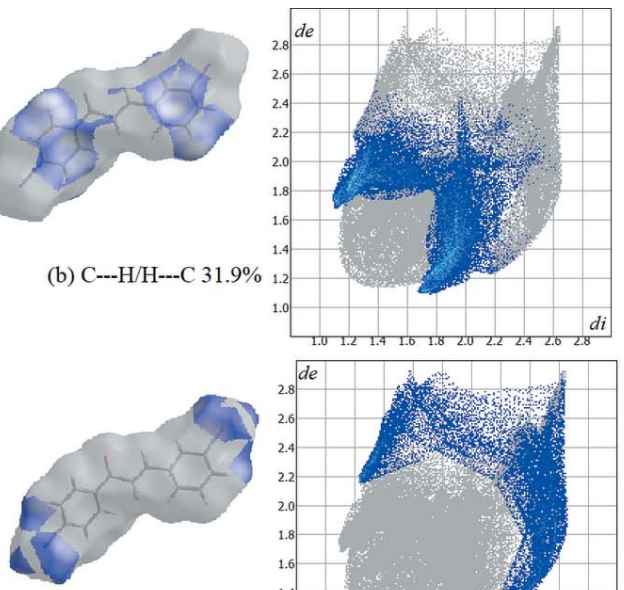

(d) I---H/H---I $18.4 \%$
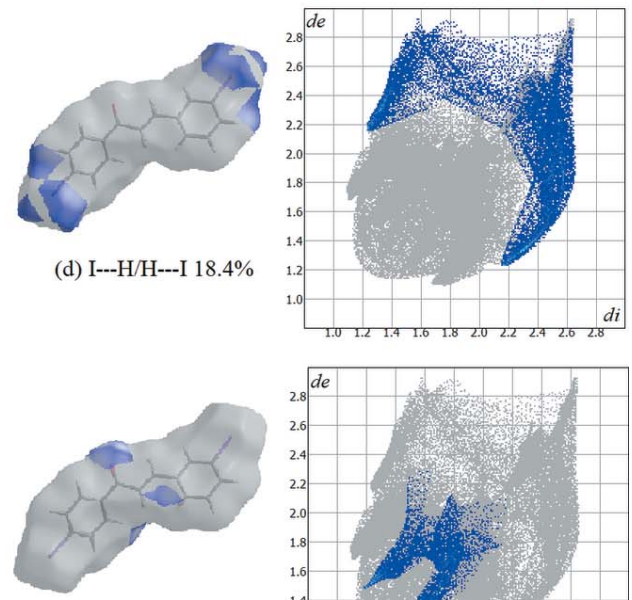

(f) $\mathrm{O}---\mathrm{H} / \mathrm{H}---\mathrm{O} 8.1 \%$

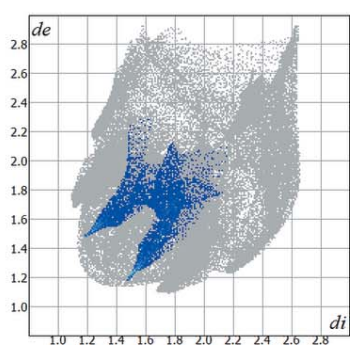

Figure 5

Hirshfeld surfaces and fingerprint plots showing percentage of contacts of $(a)$ all interactions, $(b) \mathrm{C} \cdots \mathrm{H} / \mathrm{H} \cdots \mathrm{C},(c) \mathrm{H} \cdots \mathrm{H},(d) \mathrm{I} \cdots \mathrm{H} / \mathrm{H} \cdots \mathrm{I},(e) \mathrm{I} \cdots \mathrm{I}$ and $(f) \mathrm{O} \cdots \mathrm{H} / \mathrm{H} \cdots \mathrm{O}$. interactions. 
Table 2

Experimental details.

\begin{tabular}{|c|c|}
\hline \multicolumn{2}{|l|}{ Crystal data } \\
\hline Chemical formula & $\mathrm{C}_{15} \mathrm{H}_{10} \mathrm{I}_{2} \mathrm{O}$ \\
\hline$M_{\mathrm{r}}$ & 460.03 \\
\hline Crystal system, space group & Monoclinic, $P 2_{1} / c$ \\
\hline Temperature $(\mathrm{K})$ & 200 \\
\hline$a, b, c(\AA)$ & 7.2650 (7), $32.864(3), 5.8446(6)$ \\
\hline$\beta\left(^{\circ}\right)$ & $92.277(2)$ \\
\hline$V\left(\AA^{3}\right)$ & $1394.3(2)$ \\
\hline$Z$ & 4 \\
\hline Radiation type & Мo $K \alpha$ \\
\hline$\mu\left(\mathrm{mm}^{-1}\right)$ & 4.50 \\
\hline Crystal size $(\mathrm{mm})$ & $0.57 \times 0.29 \times 0.08$ \\
\hline \multicolumn{2}{|l|}{ Data collection } \\
\hline Diffractometer & $\begin{array}{l}\text { Bruker APEXII kappa CCD area } \\
\text { detector }\end{array}$ \\
\hline Absorption correction & $\begin{array}{l}\text { Numerical (SADABS; Bruker, } \\
\text { 2016) }\end{array}$ \\
\hline$T_{\min }, T_{\max }$ & $0.065,0.189$ \\
\hline $\begin{array}{l}\text { No. of measured, independent and } \\
\text { observed }[I>2 \sigma(I)] \text { reflections }\end{array}$ & $18346,3215,2960$ \\
\hline$R_{\text {int }}$ & 0.021 \\
\hline$(\sin \theta / \lambda)_{\max }\left(\AA^{-1}\right)$ & 0.650 \\
\hline \multicolumn{2}{|l|}{ Refinement } \\
\hline$R\left[F^{2}>2 \sigma\left(F^{2}\right)\right], w R\left(F^{2}\right), S$ & $0.037,0.073,1.27$ \\
\hline No. of reflections & 3215 \\
\hline No. of parameters & 164 \\
\hline H-atom treatment & $\mathrm{H}$-atom parameters constrained \\
\hline$\Delta \rho_{\max }, \Delta \rho_{\min }\left(\mathrm{e} \AA^{-3}\right)$ & $1.14,-1.31$ \\
\hline
\end{tabular}

Computer programs: SAINT (Bruker, 2016), SHELXT (Sheldrick, 2015a), SHELXL2018 (Sheldrick, 2015b) and OLEX2 (Dolomanov et al., 2009).

make the largest contribution (31.9\%) and show a pair of spikes at $d_{\mathrm{e}}+d_{\mathrm{i}}=\sim 2.8 \AA$, representative of intermolecular $\mathrm{C}-\mathrm{H} \cdots \pi$ interactions. The $\mathrm{O} \cdots \mathrm{H} / \mathrm{H} \cdots \mathrm{O}$ plot also contains a pair of spikes at $d_{\mathrm{e}}+d_{\mathrm{i}}=\sim 2.7 \AA$ [Fig. 5(f)]. The negligible contributions from other contacts, not included in Fig. 5, are as follows: $\mathrm{C} \cdots \mathrm{C}(3.1 \%), \mathrm{C} \cdots \mathrm{O} / \mathrm{O} \cdots \mathrm{C}(2.1 \%)$ and $\mathrm{C} \cdots \mathrm{I} / \mathrm{I} \cdots \mathrm{C}$ $(0.5 \%)$.

\section{Database survey}

A survey of the Cambridge Structural Database (CSD; Groom et al., 2016) showed that existing similar structures include (2E)-1-(4-bromophenyl)-3-(4-fluorophenyl)prop-2-en-1-one (refcode NURCIN; Dutkiewicz et al., 2010), 1-(4-bromophenyl)-3-(4-chlorophenyl)prop-2-en-1-one (LEPYIP; Yang et al., 2006), 1,3-bis(4-bromophenyl)prop-2-en-1-one (LEHROG; Ng et al., 2006), (E)-1-(4-bromophenyl)-3-(4iodophenyl)prop-2-en-1-one (IWALAV; Zainuri et al., 2017) and 3-(3-bromophenyl)-1-(4-bromophenyl)prop-2-en-1-one (ODEDEH; Teh et al., 2006). Four compounds (NURCIN, LEPYIP, LEHROG and IWALAV) contain only parasubstituted rings. Within these structures, halogen bonds exist only between bromine and iodine species, and never between equivalent halogens. 3-(3-Bromophenyl)-1-(4-bromophenyl)prop-2-en-1-one contains one meta-substituted ring and one para-substituted ring: each halogen bond exists between rings with the same substitution, either para-para or meta-meta, as seen in the title compound.

\section{Synthesis and crystallization}

4'-Iodoacetophenone $\quad(0.773 \mathrm{~g}, \quad 3.14 \mathrm{mmol}), \quad 3$-iodobenzaldehyde $(0.697 \mathrm{~g}, 3.00)$, anhydrous zinc chloride $(0.615 \mathrm{~g}$, $4.51 \mathrm{mmol})$ and absolute ethanol $(1.5 \mathrm{ml})$ were added to a microwave vessel with a stir bar. Using a microwave reactor, the reaction mixture was heated to $468 \mathrm{~K}$ for 15 minutes. Upon cooling the reaction, yellowish solids were collected by vacuum filtration and washed with $95 \%$ ethanol. The resulting solid was recrystallized from $95 \%$ ethanol $(0.603 \mathrm{~g}, 44 \%$ yield, yellow crystals, m.p. 442.5-443.7 K). ${ }^{1} \mathrm{H}$ NMR $(400 \mathrm{MHz}$, DMSO- $d_{6}$, referenced to TMS): $\delta(\mathrm{ppm}) 8.37(1 \mathrm{H}, s), 8.0-7.94$ $(5 \mathrm{H}, m), 7.88(1 \mathrm{H}, d, J=8 \mathrm{~Hz}), 7.81(1 \mathrm{H}, d, J=8 \mathrm{~Hz}), 7.68(1 \mathrm{H}$, $d, J=16 \mathrm{~Hz}), 7.26(1 \mathrm{H}, t, J=8 \mathrm{~Hz}) .{ }^{13} \mathrm{C}$ NMR $(100 \mathrm{MHz}$, DMSO- $d_{6}$, referenced to solvent, $\left.39.52 \mathrm{ppm}\right): \delta(\mathrm{ppm}) 188.44$, $142.75,139.04,137.72,136.95,136.66,136.61,130.89$, 130.38, $128.73,122.81,102.14,95.57$. Single crystals suitable for X-ray diffraction were obtained by the slow evaporation technique from an acetone solution at room temperature.

\section{Refinement}

Crystal data, data collection and structure refinement details are summarized in Table 2. $\mathrm{H}$ atoms were positioned geometrically $(\mathrm{C}-\mathrm{H}=0.95 \AA)$ and refined using a riding model with $U_{\text {iso }}(\mathrm{H})=1.2 U_{\text {eq }}(\mathrm{C})$.

\section{Acknowledgements}

GU co-authors thank E. Mermann-Jozwiak, J. Hazen, S. Economu, M. Fellin, B. Hendricks, R. Meehan, \& G. Warren for their assistance, as well as the Howard Hughes Medical Institute through its Undergraduate Science Education Program for supporting equipment acquisition.

\section{Funding information}

UoB co-authors acknowledge the Engineering and Physical Sciences Research Council UK (grant EP/G036780/1) and the Centre for Doctoral Training in Condensed Matter Physics for project funding. SRH and JP acknowledge MagnaPharm, a collaborative research project funded by the European Union's Horizon 2020 Research and Innovation programme (grant No. 736899).

\section{References}

Bruker (2016). SAINT and SADABS. Bruker Analytical X-ray Instruments Inc., Madison, WI, USA.

Dolomanov, O. V., Bourhis, L. J., Gildea, R. J., Howard, J. A. K. \& Puschmann, H. (2009). J. Appl. Cryst. 42, 339-341.

D'silva, E. D., Podagatlapalli, G. K., Rao, S. V., Rao, D. N. \& Dharmaprakash, S. M. (2011). Cryst. Growth Des. 11, 5326-5369.

Dutkiewicz, G., Veena, K., Narayana, B., Yathirajan, H. S. \& Kubicki, M. (2010). Acta Cryst. E66, o1243-01244.

Groom, C. R., Bruno, I. J., Lightfoot, M. P. \& Ward, S. C. (2016). Acta Cryst. B72, 171-179.

Ng, S.-L., Shettigar, V., Razak, I. A., Fun, H.-K., Patil, P. S. \& Dharmaprakash, S. M. (2006). Acta Cryst. E62, o1421-o1423.

Sheldrick, G. M. (2015a). Acta Cryst. A71, 3-8.

Sheldrick, G. M. (2015b). Acta Cryst. C71, 3-8. 
Shetty, T. C. S., Chidan Kumar, C. S., Gagan Patel, K. N., Chia, T. S., Dharmaprakash, S. M., Ramasami, P., Umar, Y., Chandraju, S. \& Quah, C. K. (2017). J. Mol. Struct. 1143, 306-317.

Shetty, T. C. S., Raghavendra, S., Chidan Kumar, C. S. \& Dharmaprakash, S. M. (2016). Appl. Phys. B, 122, 205.

Teh, J. B.-J., Patil, P. S., Fun, H.-K., Razak, I. A. \& Dharmaprakash, S. M. (2006). Acta Cryst. E62, o2399-o2400.

Turner, M. J., McKinnon, J. J., Wolff, S. K., Grimwood, D. J., Spackman, P. R., Jayatilaka, D. \& Spackman, M. A. (2017). CrystalExplorer. Version 17. University of Western Australia.
Venkatesan, P., Thamotharan, S., Ilangovan, A., Liang, H. \& Sundius, T. (2016). Spectrochim. Acta Part A, 153, 625-636.

Yang, W., Wang, L. \& Zhang, D. (2006). J. Chem. Crystallogr. 36, 195198.

Zainuri, D. A., Arshad, S., Khalib, N. C., Razak, I. A., Pillai, R. R., Sulaiman, S. F., Hashim, N. S., Ooi, K. L., Armaković, S., Armaković, S. J., Panicker, C. Y. \& Van Alsenoy, C. (2017). J. Mol. Struct. 1128, 520-533. 


\section{supporting information}

Acta Cryst. (2020). E76, 72-76 [https://doi.org/10.1107/S2056989019016402]

Crystal structure and Hirshfeld surface analysis of (E)-3-(3-iodophenyl)-1-(4iodophenyl)prop-2-en-1-one

Kieran J. Spruce, Charlie L. Hall, Jason Potticary, Natalie E. Pridmore, Matthew E. Cremeens, Gemma D. D'ambruoso, Masaomi Matsumoto, Gabrielle I. Warren, Stephen D. Warren and Simon R. Hall

Computing details

Data collection: SAINT (Bruker, 2016); cell refinement: SAINT (Bruker, 2016); data reduction: SAINT (Bruker, 2016); program(s) used to solve structure: SHELXT (Sheldrick, 2015a); program(s) used to refine structure: SHELXL2018 (Sheldrick, 2015b); molecular graphics: OLEX2 (Dolomanov et al., 2009); software used to prepare material for publication: OLEX2 (Dolomanov et al., 2009).

(E)-3-(3-lodophenyl)-1-(4-iodophenyl) prop-2-en-1-one

Crystal data

$\mathrm{C}_{15} \mathrm{H}_{10} \mathrm{I}_{2} \mathrm{O}$

$M_{r}=460.03$

Monoclinic, $P 2_{1} / c$

$a=7.2650$ (7) $\AA$

$b=32.864(3) \AA$

$c=5.8446(6) \AA$

$\beta=92.277(2)^{\circ}$

$V=1394.3(2) \AA^{3}$

$Z=4$

\section{Data collection}

Bruker APEXII kappa CCD area detector diffractometer

Radiation source: fine-focus sealed tube Graphite monochromator $\varphi$ and $\omega$ scans

Absorption correction: numerical

(SADABS; Bruker, 2016)

$T_{\min }=0.065, T_{\max }=0.189$

Refinement

Refinement on $F^{2}$

Least-squares matrix: full

$R\left[F^{2}>2 \sigma\left(F^{2}\right)\right]=0.037$

$w R\left(F^{2}\right)=0.073$

$S=1.27$

3215 reflections
$F(000)=856$

$D_{\mathrm{x}}=2.191 \mathrm{Mg} \mathrm{m}^{-3}$

Mo $K \alpha$ radiation, $\lambda=0.71073 \AA$

Cell parameters from 8513 reflections

$\theta=2.5-27.5^{\circ}$

$\mu=4.50 \mathrm{~mm}^{-1}$

$T=200 \mathrm{~K}$

Plate, clear colourless

$0.57 \times 0.29 \times 0.08 \mathrm{~mm}$

18346 measured reflections

3215 independent reflections

2960 reflections with $I>2 \sigma(I)$

$R_{\text {int }}=0.021$

$\theta_{\max }=27.5^{\circ}, \theta_{\min }=2.5^{\circ}$

$h=-9 \rightarrow 9$

$k=-42 \rightarrow 37$

$l=-7 \rightarrow 7$

164 parameters

0 restraints

Primary atom site location: iterative

Hydrogen site location: inferred from neighbouring sites

$\mathrm{H}$-atom parameters constrained 
$w=1 /\left[\sigma^{2}\left(F_{\mathrm{o}}^{2}\right)+(0.0034 P)^{2}+6.4729 P\right]$

where $P=\left(F_{\mathrm{o}}^{2}+2 F_{\mathrm{c}}^{2}\right) / 3$

$(\Delta / \sigma)_{\max }=0.001$

$\Delta \rho_{\max }=1.14$ e $\AA^{-3}$

$\Delta \rho_{\min }=-1.30$ e $\AA^{-3}$

Special details

Geometry. All esds (except the esd in the dihedral angle between two 1.s. planes) are estimated using the full covariance matrix. The cell esds are taken into account individually in the estimation of esds in distances, angles and torsion angles; correlations between esds in cell parameters are only used when they are defined by crystal symmetry. An approximate (isotropic) treatment of cell esds is used for estimating esds involving l.s. planes.

Fractional atomic coordinates and isotropic or equivalent isotropic displacement parameters $\left(\AA^{2}\right)$
Extinction correction: SHELXL2018

(Sheldrick, 2015b),

$\mathrm{Fc}^{*}=\mathrm{kFc}\left[1+0.001 \times \mathrm{xc}^{2} \lambda^{3} / \sin (2 \theta)\right]^{-1 / 4}$

Extinction coefficient: 0.00062 (7)

\begin{tabular}{|c|c|c|c|c|}
\hline & $x$ & $y$ & $z$ & $U_{\text {iso }} * / U_{\text {eq }}$ \\
\hline $\mathrm{C} 4$ & $0.8336(6)$ & $0.43230(13)$ & $0.5707(7)$ & $0.0250(9)$ \\
\hline C9 & $0.9114(6)$ & $0.42521(14)$ & $0.7892(8)$ & $0.0278(9)$ \\
\hline H9 & 0.939830 & 0.447424 & 0.888449 & $0.033^{*}$ \\
\hline $\mathrm{C} 8$ & $0.9474(6)$ & 0.38548 (14) & $0.8617(8)$ & $0.0289(9)$ \\
\hline $\mathrm{H} 8$ & 1.003866 & 0.380553 & 1.008533 & $0.035^{*}$ \\
\hline $\mathrm{C} 7$ & $0.9004(6)$ & $0.35322(14)$ & $0.7183(8)$ & $0.0292(9)$ \\
\hline C6 & $0.8265(6)$ & 0.35974 (14) & $0.4992(8)$ & $0.0298(10)$ \\
\hline H6 & 0.797488 & 0.337429 & 0.400879 & $0.036^{*}$ \\
\hline $\mathrm{C} 5$ & $0.7955(6)$ & 0.39954 (14) & $0.4252(8)$ & $0.0285(9)$ \\
\hline H5 & 0.747935 & 0.404397 & 0.273958 & $0.034^{*}$ \\
\hline $\mathrm{C} 1$ & $0.7892(6)$ & 0.47421 (14) & $0.4874(8)$ & $0.0284(9)$ \\
\hline $\mathrm{C} 2$ & $0.7447(6)$ & 0.50560 (14) & $0.6605(8)$ & 0.0294 (9) \\
\hline $\mathrm{H} 2$ & 0.729579 & 0.497837 & 0.815221 & $0.035^{*}$ \\
\hline $\mathrm{C} 3$ & $0.7258(6)$ & $0.54438(13)$ & $0.5999(8)$ & 0.0268 (9) \\
\hline H3 & 0.749373 & 0.550781 & 0.445302 & $0.032 *$ \\
\hline $\mathrm{C} 10$ & $0.6729(6)$ & $0.57825(13)$ & $0.7450(7)$ & $0.0251(9)$ \\
\hline C11 & $0.6878(6)$ & $0.61791(13)$ & $0.6589(8)$ & $0.0273(9)$ \\
\hline H11 & 0.740192 & 0.622248 & 0.514565 & $0.033^{*}$ \\
\hline $\mathrm{C} 12$ & $0.6264(6)$ & $0.65086(13)$ & $0.7836(8)$ & $0.0289(9)$ \\
\hline $\mathrm{C} 13$ & $0.5540(6)$ & $0.64548(15)$ & $0.9989(8)$ & $0.0322(10)$ \\
\hline H13 & 0.513741 & 0.668101 & 1.084900 & $0.039^{*}$ \\
\hline C14 & $0.5424(6)$ & 0.60607 (15) & $1.0845(8)$ & $0.0311(10)$ \\
\hline H14 & 0.493446 & 0.601952 & 1.230991 & $0.037^{*}$ \\
\hline $\mathrm{C} 15$ & $0.6003(6)$ & $0.57271(14)$ & $0.9618(8)$ & $0.0280(9)$ \\
\hline H15 & 0.590951 & 0.546131 & 1.024224 & $0.034^{*}$ \\
\hline I1 & $0.92866(6)$ & $0.29371(2)$ & $0.84509(7)$ & $0.04484(12)$ \\
\hline $\mathrm{I} 2$ & $0.63173(6)$ & $0.70888(2)$ & $0.63477(6)$ & $0.04434(12)$ \\
\hline $\mathrm{O} 1$ & $0.7830(5)$ & $0.48192(10)$ & $0.2821(6)$ & $0.0376(8)$ \\
\hline
\end{tabular}

Atomic displacement parameters $\left(\AA^{2}\right)$

\begin{tabular}{lllllll}
\hline & $U^{11}$ & $U^{22}$ & $U^{33}$ & $U^{12}$ & $U^{13}$ & $U^{23}$ \\
\hline C4 & $0.022(2)$ & $0.027(2)$ & $0.026(2)$ & $0.0011(17)$ & $0.0023(16)$ & $0.0009(17)$ \\
C9 & $0.027(2)$ & $0.028(2)$ & $0.028(2)$ & $-0.0020(18)$ & $-0.0031(18)$ & $-0.0029(17)$
\end{tabular}




$\begin{array}{lllllll}\text { C8 } & 0.028(2) & 0.034(2) & 0.024(2) & 0.0032(19) & -0.0031(17) & 0.0017(18) \\ \text { C7 } & 0.027(2) & 0.026(2) & 0.034(2) & 0.0048(18) & 0.0022(18) & 0.0026(18) \\ \text { C6 } & 0.029(2) & 0.029(2) & 0.031(2) & 0.0003(18) & -0.0001(18) & -0.0077(18) \\ \text { C5 } & 0.028(2) & 0.033(2) & 0.024(2) & 0.0036(18) & -0.0010(17) & -0.0009(17) \\ \text { C1 } & 0.023(2) & 0.031(2) & 0.031(2) & 0.0005(17) & 0.0008(18) & 0.0024(18) \\ \text { C2 } & 0.033(2) & 0.028(2) & 0.027(2) & -0.0004(18) & 0.0022(18) & 0.0019(17) \\ \text { C3 } & 0.026(2) & 0.029(2) & 0.026(2) & -0.0005(18) & 0.0003(17) & 0.0016(17) \\ \text { C10 } & 0.021(2) & 0.030(2) & 0.025(2) & -0.0016(17) & -0.0045(16) & 0.0015(17) \\ \text { C11 } & 0.027(2) & 0.029(2) & 0.026(2) & -0.0048(18) & -0.0012(17) & 0.0022(17) \\ \text { C12 } & 0.031(2) & 0.024(2) & 0.031(2) & -0.0037(18) & -0.0077(18) & 0.0016(17) \\ \text { C13 } & 0.028(2) & 0.035(2) & 0.033(2) & 0.0014(19) & -0.0018(19) & -0.0073(19) \\ \text { C14 } & 0.028(2) & 0.041(3) & 0.024(2) & -0.002(2) & -0.0003(18) & -0.0015(19) \\ \text { C15 } & 0.025(2) & 0.030(2) & 0.028(2) & 0.0001(18) & -0.0048(17) & 0.0043(18) \\ \text { I1 } & 0.0555(2) & 0.02652(17) & 0.0520(2) & 0.00766(15) & -0.00426(16) & 0.00411(14) \\ \text { I2 } & 0.0618(3) & 0.02421(16) & 0.0466(2) & -0.00405(15) & -0.00256(16) & 0.00229(14) \\ \text { O1 } & 0.051(2) & 0.0340(18) & 0.0278(17) & 0.0045(16) & 0.0029(15) & 0.0051(14)\end{array}$

Geometric parameters $\left(\AA,{ }^{\circ}\right)$

\begin{tabular}{llll}
\hline $\mathrm{C} 4-\mathrm{C} 9$ & $1.395(6)$ & $\mathrm{C} 2-\mathrm{C} 3$ & $1.328(6)$ \\
$\mathrm{C} 4-\mathrm{C} 5$ & $1.393(6)$ & $\mathrm{C} 3-\mathrm{H} 3$ & 0.9500 \\
$\mathrm{C} 4-\mathrm{C} 1$ & $1.492(6)$ & $\mathrm{C} 3-\mathrm{C} 10$ & $1.460(6)$ \\
$\mathrm{C} 9-\mathrm{H} 9$ & 0.9500 & $\mathrm{C} 10-\mathrm{C} 11$ & $1.403(6)$ \\
$\mathrm{C} 9-\mathrm{C} 8$ & $1.394(6)$ & $\mathrm{C} 10-\mathrm{C} 15$ & $1.403(6)$ \\
$\mathrm{C} 8-\mathrm{H} 8$ & 0.9500 & $\mathrm{C} 11-\mathrm{H} 11$ & 0.9500 \\
$\mathrm{C} 8-\mathrm{C} 7$ & $1.386(6)$ & $\mathrm{C} 11-\mathrm{C} 12$ & $1.388(6)$ \\
$\mathrm{C} 7-\mathrm{C} 6$ & $1.386(6)$ & $\mathrm{C} 12-\mathrm{C} 13$ & $1.394(7)$ \\
$\mathrm{C} 7-\mathrm{I} 1$ & $2.099(4)$ & $\mathrm{C} 12-\mathrm{I} 2$ & $2.097(4)$ \\
$\mathrm{C} 6-\mathrm{H} 6$ & 0.9500 & $\mathrm{C} 13-\mathrm{H} 13$ & 0.9500 \\
$\mathrm{C} 6-\mathrm{C} 5$ & $1.393(6)$ & $\mathrm{C} 13-\mathrm{C} 14$ & $1.392(7)$ \\
$\mathrm{C} 5-\mathrm{H} 5$ & 0.9500 & $\mathrm{C} 14-\mathrm{H} 14$ & 0.9500 \\
$\mathrm{C} 1-\mathrm{C} 2$ & $1.490(6)$ & $\mathrm{C} 14-\mathrm{C} 15$ & $1.385(7)$ \\
$\mathrm{C} 1-\mathrm{O} 1$ & $1.225(5)$ & $\mathrm{C} 15-\mathrm{H} 15$ & 0.9500 \\
$\mathrm{C} 2-\mathrm{H} 2$ & 0.9500 & & 119.8 \\
& & & 116.4 \\
$\mathrm{C} 9-\mathrm{C} 4-\mathrm{C} 1$ & $121.8(4)$ & $\mathrm{C} 3-\mathrm{C} 2-\mathrm{H} 2$ & $127.1(4)$ \\
$\mathrm{C} 5-\mathrm{C} 4-\mathrm{C} 9$ & $119.5(4)$ & $\mathrm{C} 2-\mathrm{C} 3-\mathrm{H} 3$ & 116.4 \\
$\mathrm{C} 5-\mathrm{C} 4-\mathrm{C} 1$ & $118.6(4)$ & $\mathrm{C} 2-\mathrm{C} 3-\mathrm{C} 10$ & $118.3(4)$ \\
$\mathrm{C} 4-\mathrm{C} 9-\mathrm{H} 9$ & 120.0 & $\mathrm{C} 10-\mathrm{C} 3-\mathrm{H} 3$ & $118.8(4)$ \\
$\mathrm{C} 8-\mathrm{C} 9-\mathrm{C} 4$ & $119.9(4)$ & $\mathrm{C} 11-\mathrm{C} 10-\mathrm{C} 3$ & $122.8(4)$ \\
$\mathrm{C} 8-\mathrm{C} 9-\mathrm{H} 9$ & 120.0 & $\mathrm{C} 11-\mathrm{C} 10-\mathrm{C} 15$ & 119.8 \\
$\mathrm{C} 9-\mathrm{C} 8-\mathrm{H} 8$ & 120.2 & $\mathrm{C} 15-\mathrm{C} 10-\mathrm{C} 3$ & $120.4(4)$ \\
$\mathrm{C} 7-\mathrm{C} 8-\mathrm{C} 9$ & $119.6(4)$ & $\mathrm{C} 10-\mathrm{C} 11-\mathrm{H} 11$ & 119.8 \\
$\mathrm{C} 7-\mathrm{C} 8-\mathrm{H} 8$ & 120.2 & $\mathrm{C} 12-\mathrm{C} 11-\mathrm{C} 10$ & $121.0(4)$ \\
$\mathrm{C} 8-\mathrm{C} 7-\mathrm{I} 1$ & $118.8(3)$ & $\mathrm{C} 12-\mathrm{C} 11-\mathrm{H} 11$ & $118.7(3)$ \\
$\mathrm{C} 6-\mathrm{C} 7-\mathrm{C} 8$ & $121.2(4)$ & $\mathrm{C} 11-\mathrm{C} 12-\mathrm{C} 12-\mathrm{I} 13$ & $120.2(3)$ \\
$\mathrm{C} 6-\mathrm{C} 7-\mathrm{I} 1$ & $120.0(3)$ & $\mathrm{C} 12-\mathrm{I} 2$ & \\
$\mathrm{C} 7-\mathrm{C} 6-\mathrm{H} 6$ & 120.5 & &
\end{tabular}




\begin{tabular}{|c|c|c|c|}
\hline $\mathrm{C} 7-\mathrm{C} 6-\mathrm{C} 5$ & $118.9(4)$ & $\mathrm{C} 12-\mathrm{C} 13-\mathrm{H} 13$ & 120.9 \\
\hline $\mathrm{C} 5-\mathrm{C} 6-\mathrm{H} 6$ & 120.5 & $\mathrm{C} 14-\mathrm{C} 13-\mathrm{C} 12$ & $118.2(4)$ \\
\hline $\mathrm{C} 4-\mathrm{C} 5-\mathrm{C} 6$ & $120.7(4)$ & $\mathrm{C} 14-\mathrm{C} 13-\mathrm{H} 13$ & 120.9 \\
\hline $\mathrm{C} 4-\mathrm{C} 5-\mathrm{H} 5$ & 119.6 & $\mathrm{C} 13-\mathrm{C} 14-\mathrm{H} 14$ & 119.1 \\
\hline $\mathrm{C} 6-\mathrm{C} 5-\mathrm{H} 5$ & 119.6 & $\mathrm{C} 15-\mathrm{C} 14-\mathrm{C} 13$ & $121.8(4)$ \\
\hline $\mathrm{C} 2-\mathrm{C} 1-\mathrm{C} 4$ & $117.9(4)$ & $\mathrm{C} 15-\mathrm{C} 14-\mathrm{H} 14$ & 119.1 \\
\hline $\mathrm{O} 1-\mathrm{C} 1-\mathrm{C} 4$ & $120.6(4)$ & $\mathrm{C} 10-\mathrm{C} 15-\mathrm{H} 15$ & 120.1 \\
\hline $\mathrm{O} 1-\mathrm{C} 1-\mathrm{C} 2$ & $121.4(4)$ & $\mathrm{C} 14-\mathrm{C} 15-\mathrm{C} 10$ & $119.8(4)$ \\
\hline $\mathrm{C} 1-\mathrm{C} 2-\mathrm{H} 2$ & 119.8 & $\mathrm{C} 14-\mathrm{C} 15-\mathrm{H} 15$ & 120.1 \\
\hline $\mathrm{C} 3-\mathrm{C} 2-\mathrm{C} 1$ & $120.4(4)$ & & \\
\hline $\mathrm{C} 4-\mathrm{C} 9-\mathrm{C} 8-\mathrm{C} 7$ & $-1.9(7)$ & $\mathrm{C} 2-\mathrm{C} 3-\mathrm{C} 10-\mathrm{C} 11$ & $170.4(5)$ \\
\hline $\mathrm{C} 4-\mathrm{C} 1-\mathrm{C} 2-\mathrm{C} 3$ & $171.9(4)$ & $\mathrm{C} 2-\mathrm{C} 3-\mathrm{C} 10-\mathrm{C} 15$ & $-12.5(7)$ \\
\hline $\mathrm{C} 9-\mathrm{C} 4-\mathrm{C} 5-\mathrm{C} 6$ & $2.9(7)$ & $\mathrm{C} 3-\mathrm{C} 10-\mathrm{C} 11-\mathrm{C} 12$ & $175.2(4)$ \\
\hline $\mathrm{C} 9-\mathrm{C} 4-\mathrm{C} 1-\mathrm{C} 2$ & $-28.3(6)$ & $\mathrm{C} 3-\mathrm{C} 10-\mathrm{C} 15-\mathrm{C} 14$ & $-176.0(4)$ \\
\hline $\mathrm{C} 9-\mathrm{C} 4-\mathrm{C} 1-\mathrm{O} 1$ & $154.5(5)$ & $\mathrm{C} 10-\mathrm{C} 11-\mathrm{C} 12-\mathrm{C} 13$ & $1.9(7)$ \\
\hline $\mathrm{C} 9-\mathrm{C} 8-\mathrm{C} 7-\mathrm{C} 6$ & $3.3(7)$ & $\mathrm{C} 10-\mathrm{C} 11-\mathrm{C} 12-\mathrm{I} 2$ & $-175.0(3)$ \\
\hline $\mathrm{C} 9-\mathrm{C} 8-\mathrm{C} 7-\mathrm{I} 1$ & $-173.2(3)$ & $\mathrm{C} 11-\mathrm{C} 10-\mathrm{C} 15-\mathrm{C} 14$ & $1.0(6)$ \\
\hline $\mathrm{C} 8-\mathrm{C} 7-\mathrm{C} 6-\mathrm{C} 5$ & $-1.5(7)$ & $\mathrm{C} 11-\mathrm{C} 12-\mathrm{C} 13-\mathrm{C} 14$ & $-0.9(7)$ \\
\hline $\mathrm{C} 7-\mathrm{C} 6-\mathrm{C} 5-\mathrm{C} 4$ & $-1.6(7)$ & $\mathrm{C} 12-\mathrm{C} 13-\mathrm{C} 14-\mathrm{C} 15$ & $0.0(7)$ \\
\hline $\mathrm{C} 5-\mathrm{C} 4-\mathrm{C} 9-\mathrm{C} 8$ & $-1.1(7)$ & $\mathrm{C} 13-\mathrm{C} 14-\mathrm{C} 15-\mathrm{C} 10$ & $0.0(7)$ \\
\hline $\mathrm{C} 5-\mathrm{C} 4-\mathrm{C} 1-\mathrm{C} 2$ & $151.6(4)$ & $\mathrm{C} 15-\mathrm{C} 10-\mathrm{C} 11-\mathrm{C} 12$ & $-1.9(6)$ \\
\hline $\mathrm{C} 5-\mathrm{C} 4-\mathrm{C} 1-\mathrm{O} 1$ & $-25.6(7)$ & $\mathrm{I} 1-\mathrm{C} 7-\mathrm{C} 6-\mathrm{C} 5$ & $175.0(3)$ \\
\hline $\mathrm{C} 1-\mathrm{C} 4-\mathrm{C} 9-\mathrm{C} 8$ & $178.8(4)$ & $\mathrm{I} 2-\mathrm{C} 12-\mathrm{C} 13-\mathrm{C} 14$ & $175.9(3)$ \\
\hline $\mathrm{C} 1-\mathrm{C} 4-\mathrm{C} 5-\mathrm{C} 6$ & $-177.0(4)$ & $\mathrm{O} 1-\mathrm{C} 1-\mathrm{C} 2-\mathrm{C} 3$ & $-10.9(7)$ \\
\hline $\mathrm{C} 1-\mathrm{C} 2-\mathrm{C} 3-\mathrm{C} 10$ & $176.4(4)$ & & \\
\hline
\end{tabular}

Hydrogen-bond geometry $\left(A,{ }^{\circ}\right)$

$C g 1$ and $C g 2$ are the centroids of the $\mathrm{C} 10-\mathrm{C} 15$ and $\mathrm{C} 4-\mathrm{C} 9$ rings, respectively.

\begin{tabular}{lllll}
\hline$D-\mathrm{H} \cdots A$ & $D-\mathrm{H}$ & $\mathrm{H} \cdots A$ & $D \cdots A$ & $D-\mathrm{H} \cdots A$ \\
\hline $\mathrm{C} 5-\mathrm{H} 5 \cdots C g 1^{\mathrm{i}}$ & 0.95 & 2.78 & $3.406(5)$ & 124 \\
$\mathrm{C} 8-\mathrm{H} 8 \cdots C g 1^{\mathrm{ii}}$ & 0.95 & 2.85 & $3.491(5)$ & 126 \\
$\mathrm{C} 14-\mathrm{H} 14 \cdots C g 2^{\mathrm{iii}}$ & 0.95 & 2.77 & $3.440(5)$ & 129 \\
\hline
\end{tabular}

Symmetry codes: (i) $-x+1,-y+1,-z+1$; (ii) $-x+2,-y+1,-z+2$; (iii) $-x+1,-y+1,-z+2$. 